\title{
A Forest Fire Warning Method Based on Fire Dangerous Rating
}

\author{
Dan Wang ${ }^{1, a}$, Lei Xu ${ }^{1, b^{*}}$, Yuanyuan Zhou ${ }^{1, c}$, Zhifu Gao ${ }^{1, d}$ \\ ${ }^{1}$ School of Electronics and Information Engineering, Anhui University, Hefei, 230601, China \\ aemail: 18297955876@163.com, 'email:xulei@ahu.edu.cn, 'email:13003082873@163.com, \\ demail: llyahu@163.comDan Wang ${ }^{1, a}$
}

Keywords: forest fire; WSN; multilevel data fusion; fire dangerous rating; fire warning

\begin{abstract}
The importance of forest fire detecting was up to the destructive of forest fire, which can destroy resource safety and threaten human-living environment. The traditional forest fire monitoring and early warning methods are manly based on ground patrolling, watching tower, aerial patrolling, long-distance video monitoring, satellite monitoring, which are often inclined to miss the best time to extinguish the fire. This paper illustrates a real-time forest fire monitoring method based on the technology of WSN (wireless sensor network) and forest fire dangerous rating in comparison to the deficiency of traditional forest fire monitoring measures. Through the comprehensive analysis of three respects of network topology, energy saving as well as being stable and reliable, this paper brings forward the hardware design of sensor network node and network protocols suitable for forest monitoring. The designed system can conduct effective monitoring of forest environment. This paper envisages the deployment of Wireless Sensor Networks by using a multilevel data fusion to enhance the performance of the early fire detection process.
\end{abstract}

\section{Introduction}

In recent years WSN has been more and more widely used in diverse non-deterministic environments where sensors are being employed in many practical applications that require complex data sampling, analysis and a system integration framework [1]. While the forest is seen as one of the most important and indispensable resources, furthermore, as the protector of the ecological balance of the earth, forest fires often start unnoticed and spread very quickly, causing millions of dollars in damage and claiming many human lives every year [2]. At present, traditional forest fire monitoring measures mainly contain artificial ground patrolling, man watching tower in China [1]. But there are a lot of deficiencies, such as, limited application and unsatisfied monitoring results. So it's necessary to bring in a new method for effective supplements. Monitoring the fire air and ground to achieve full three-dimensional monitoring is the development trend of forest fire monitoring [3].

Many researches on forest fire detection have been conducted. Most of them make use of temperature humidity and smoke sensors [4]. Besides, unmanned aerial cruise or satellite remote sensing images are frequently used for forest fire detection and monitoring in America [5], which is incapable of real-time detection for the surrounding cycle period and the low resolution of satellite images. A system for wildfire monitoring using a wireless sensor network (WSN) that collects temperature relative humidity and barometric pressure is proposed [6]. A forest fire detection system is raised based on multi-sensor technology and neural networks [7]. The monitoring parameters include environmental temperature, $\mathrm{CO}$ and smoke density. However, there are many weaknesses; for example, most of those systems require the use of training data. This paper demonstrates an integration of multi-sensor, WSN and multilevel data fusion for detecting, monitoring and predicting forest fire. In the case of fire detection one requires measurements from clusters nodes that are deployed in the area and clusters heads which monitor the same area from a distance.

In the multilevel hierarchical clustering protocol, temperature, humidity and flame are monitored by the clusters sub-nodes, while temperature, humidity, flame, light, rain and smoke are supervised by clusters heads. The application of a multilevel fusion process assists in the direction of reducing 
false warning rates while satisfying the early fire detection requirement, contrary to the previous forest fire monitoring methods.

\section{Structure of forest fire warning method}

This paper designs a forest fire warning method based on fire dangerous rating. On the condition of forest fire, the fire warning system is able to monitor and forecast its rating, which integrates sensor technology, wireless communications and data fusion. The system designed by this paper is a complex integrated system, which is multi-level. Each level executes a specific set of functions, and neighboring level interactive to their common interface so that all data transmissions are reliable and safe. The schematic of the produce of forest fire dangerous rating is shown as figure.1. The structure of the forest fire warning mainly contains three parts: cluster sub-nodes level, cluster head nodes level, graphical server interface.

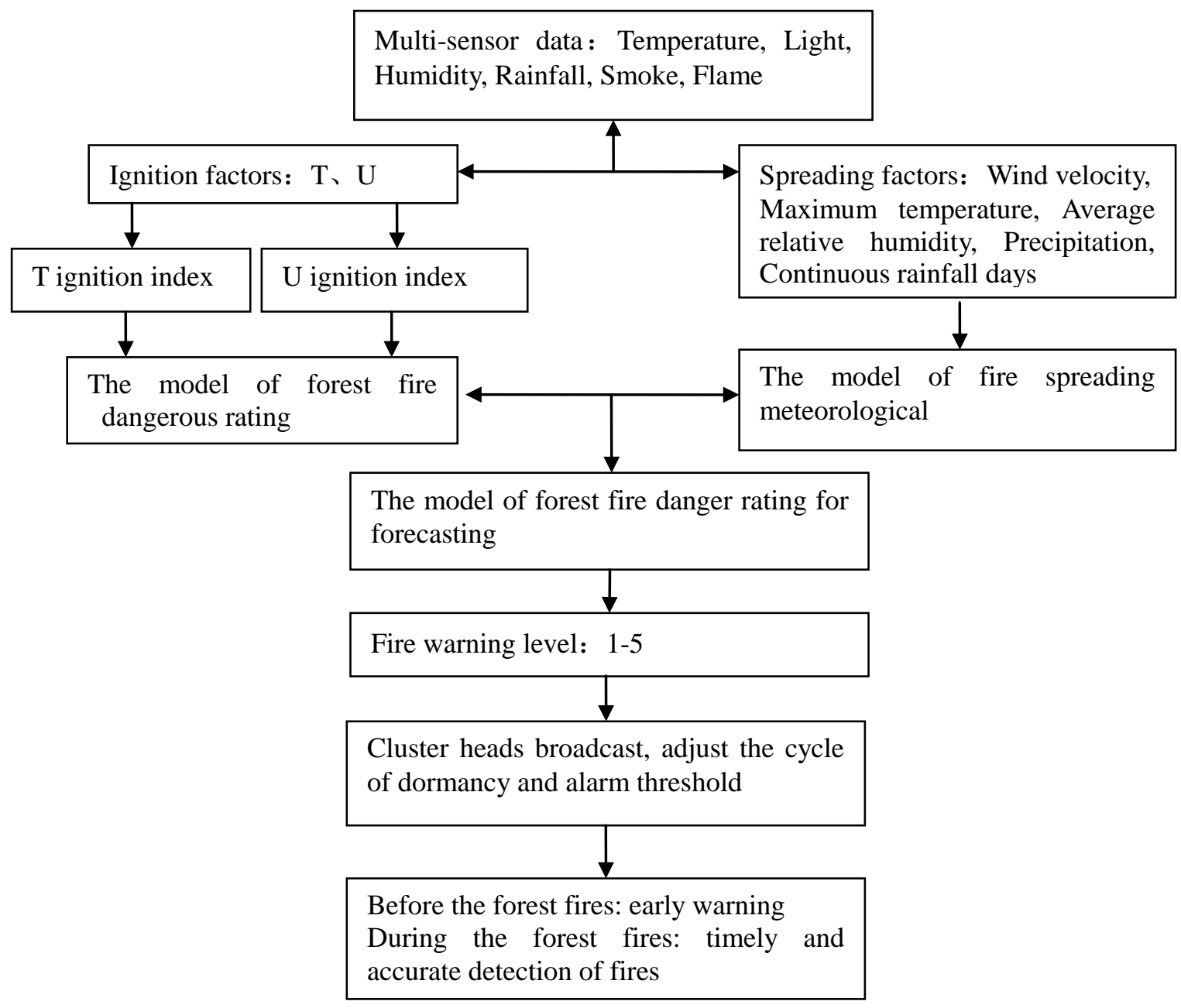

Figure.1. The workflow of forest fire warning method

\section{A. Cluster sub-nodes level}

The functions of the cluster sub-nodes level is the acquisition of the forest ignition factors that are relevant to the assessment of a natural fire. In the coverage area three kinds of sensors are deployed: Temperature sensors, humidity sensors and flame sensors. The cluster sub-nodes that are energy efficient transmit the original data to the cluster head nodes via appropriate routing protocols.

\section{B. Cluster head nodes level}

The cluster head nodes control an area of deployed cluster sub-nodes and perform the fist level data fusion algorithms for fire detection. The cluster head nodes level resides between the cluster sub-nodes level and graphical server as depicted in Figure.2. The main modules are described in the 
following paragraphs.

a. Communication module, which is responsible for the communication between the cluster sub-nodes level and graphical server.

b. Data base module, which receives monitoring data transmitted by the cluster sub-nodes. Besides, it stores historical data, the identification of each cluster sub-nodes and their locations.

c. Data fusion module, which assesses monitoring data and determines whether there is a fire in the area. Furthermore, it estimates fire dangerous rating and location of the fire.

The topology of the multilevel cluster controlled by the cluster head nodes plays a significance role in energy saving and the data fusion process. The density of nodes is determined by the required accuracy of fire location estimation.

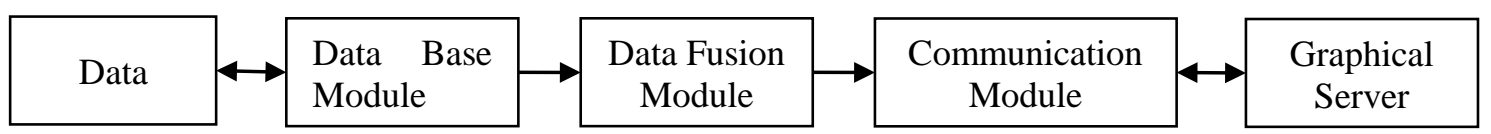

Figure.2. The architecture of cluster head nodes

\section{Graphical server interface}

The graphical server is largely based on the graphical interface and SQL server where the fire dangerous rating is produced using additional weather data such as rainfall, wind speed and direction. Mathematical environmental models are established so as to acquire an accurate result of the fire before the fire spreads. The graphical server is able to reconfigure parameters to adapt to the dynamic condition of the observed fire in order to gain the accurate monitoring.

The main functionalities of graphical server are:

a. Receive and store monitoring data from the area of WSN.

b. Perform the second level data fusion to assess the rating of fire danger.

c. Trigger a warning in case of forest fire.

\section{Multilevel Data Fusion}

The monitoring data from cluster sub-nodes are processed in the first level data fusion so as to detect any significant changes of forest fire factors, which, as a result, generate the various fire danger indices and rating. If a change in a certain monitoring fire factor is detected, it reflects on a relevant index. Such procedure is executed for each fire factor in the data fusion to calculate the probability of fire. The probabilities from first level data fusion combined with additional weather data are provided to the second level data fusion in order to make a safe decision about fire occurrence. Figure.1. depicts the workflow of forest fire warning method as it is present in the following paragraphs.

\section{A. First level fusion}

Through spot investigation in the area of forest fire as well as the experience of forestry and meteorological departments on fire prevention, we select the maximum temperature and relative humidity as main factors of forest fire. The probability of ignition is closely related to the changes of these factors. When the maximum temperature is above the average and the minimum relative humidity below the average, it entered the forest fireproof period, which the forest is the most easy to catch fire. We use Tmax refer to maximum temperature, max refer to the average of maximum temperature. Meteorological factors, temperature and relative humidity, corresponding to different level of forest fire.

$$
\triangle \mathrm{T}=\mathrm{Tmax}-\max
$$

It can gain TU index from statistical data, which is calculated by Equation 2. Let T denote $\triangle \mathrm{T}$ corresponding to ignition index. Let $\mathrm{U}$ denote the minimum relative humidity corresponding to ignition index.

$$
\mathrm{PU}=(\mathrm{T}+\mathrm{U}) / 2
$$




\section{B. Second level fusion}

We select the wind speed, the average temperature, total precipitation, the longest consecutive dry days, and the average relative humidity as a forest fire spread factor. Considering contribution of all forest fire factors to the spread of forest fires is different, we set different empirical coefficient of every forest fire factor. Forest fire spread level threshold is calculated by Equation 3, which is combined with years of experience in forest fire prevention so as to divide standards of forest fire spread grades.

$$
\mathrm{Y}=0.5 \mathrm{X}_{1}+0.15 \mathrm{X}_{2}-0.14 \mathrm{X}_{3}+0.26 \mathrm{X}_{4}-0.05 \mathrm{X}_{5}
$$

Where: $\mathrm{X}_{1}$ is the wind speed; $\mathrm{X}_{2}$ is the average air temperature; $\mathrm{X}_{3}$ is precipitation; $\mathrm{X}_{4}$ consecutive dry days; $\mathrm{X}_{5}$ is the average relative humidity.

The forest fire danger rating index $\mathrm{W}$ is calculated by the equation 4 is use $\mathrm{W}$ index search operator to obtain forest fire danger rating DJ.

$$
\mathrm{W}=0.6 \mathrm{Z}+0.4 \mathrm{M}
$$

Where: $\mathrm{Z}$ to forest fire weather rating; $\mathrm{M}$ forest fire spreading weather rating.

\section{Graphical server interface}

The algorithms of multilevel data fusion recognize whether a change of fire factors is a fire or a wrong alarm. In the former case graphical server interface initiates a simulation, which is based on a different set of input parameters. Providing the system with data about the topography, forest fire factors and the types of forest fuel, the evolution can be calculated accurately. The visualization of the graphical server interface is depicted in Figure.3.

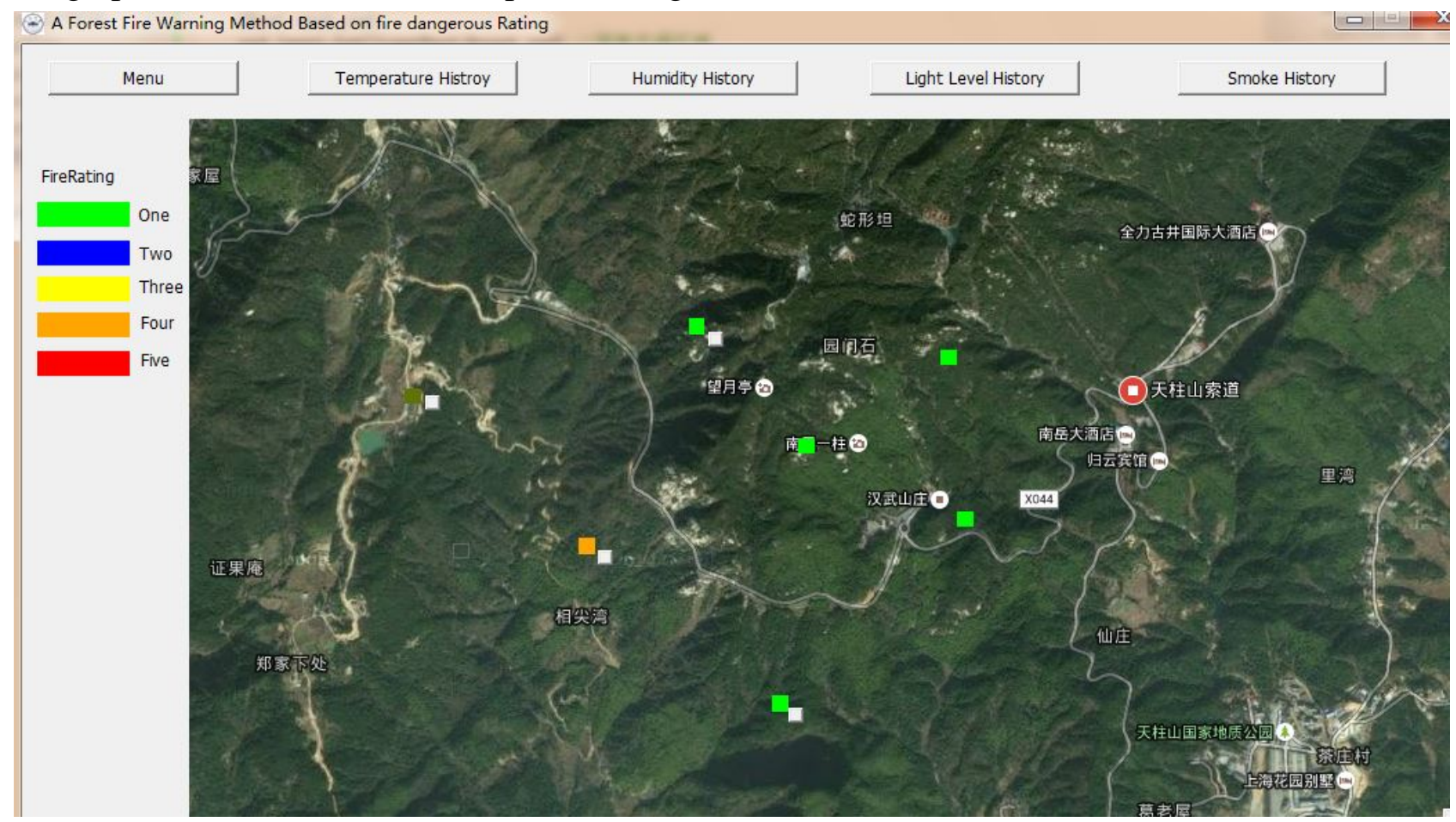

Figure.3. the graphical server interface

\section{Conclusions}

In this paper a forest fire warning method is proposed, based on fire dangerous rating. The data are collected through WSN. The multilevel data fusion has been adopted to deal with the various types of multi-sensors with high accuracy. Multi-sensor data are processed in the two levels of data fusion, which improves the reliability and accuracy in forest fire monitoring. On the first level, the statistical behavior of monitoring data is constantly assessed. On the second level, a forest fire model is built so as to combine the indications coming from the first level with the additional 
weather data. As a future work, we propose the enhancement of the implemented algorithms with alternative combination rules, and the adoption of the Fuzzy Set theory to deal with uncertainty, imprecision and incompleteness of the underlying data.

\section{Acknowledgement}

The work was supported by the Undergraduate Innovation and Entrepreneurship Training Program of Anhui University (No. 201410357044).

\section{References}

[1] Gao, Zhifu, Linsheng Huang. A Forest Fire Monitoring and Early Warning System Based on the Technology of Multi-sensor and Multilevel Data Fusion [C]. Procedings of ICECEE 2015 (2015). 2352-538x

[2] Mal-Sarkar S, Sikder I U, Konangi V K. Application of wireless sensor networks in forest fire detection under uncertainty[C].2010 13th International Conference on Computer and Information Technology (ICCIT). 2010.

[3] S.I. Stewart, V.C. Radeloff, R.B. Hammer and T.J. Hawbaker, Defining the Wildland Urban Interface, Journal of Forestry,2007, 105:201-207

[4] Z. Q. Hao and Z. J. Zhang, Two Centralized Energy-Efficient Deployment Algorithms for Mobile Nodes in a Mixed Wireless Sensor Network, Journal of Computers, vol. 24, no. 4, (2014), pp. 32-43.

[5] L. Yu, N. Wang and X. Meng, Real-time forest fire detection with wireless sensor networks, Wireless Communications, Networking and Mobile Computing, 2005. Proceedings. 2005 International Conference on IEEE, vol. 2, (2005), pp. 1214-1217.

[6] D.M. Doolin and N. Sitar, Wireless sensors for wildfire monitoring, Smart Structures and Materials, vol. 5765, March 2005.

[7] Sekkas, Odysseas, Stathes Hadjiefthymiades, and Evangelos Zervas. A multi-level data fusion approach for early fire detection. Intelligent Networking and Collaborative Systems (INCOS), 2010 2nd International Conference on. IEEE, 2010. 